ANNALES

POLONICI MATHEMATICI

$82.3(2003)$

\title{
The set of recurrent points of a continuous self-map on compact metric spaces and strong chaos
}

by Lidong Wang (Dalian), Gongfu Liao (Changchun), Zhizhi Chen (Siping) and Xiaodong DuAn (Dalian)

\begin{abstract}
We discuss the existence of an uncountable strongly chaotic set of a continuous self-map on a compact metric space. It is proved that if a continuous self-map on a compact metric space has a regular shift invariant set then it has an uncountable strongly chaotic set in which each point is recurrent, but is not almost periodic.
\end{abstract}

1. Introduction. Throughout this paper, $X$ will denote a compact metric space with metric $d$, and $I$ is the closed interval $[0,1]$.

For a continuous map $f: X \rightarrow X$, we will denote the set of almost periodic points and of recurrent points of $f$ by $A(f)$ and $R(f)$ respectively, with the usual definitions; $f^{n}$ will denote the $n$-fold iterate of $f$.

For $x, y$ in $X$, any real number $t$ and positive integer $n$, let

$$
\xi_{n}(f, x, y, t)=\#\left\{i \mid d\left(f^{i}(x), f^{i}(y)\right)<t, 1 \leq i \leq n\right\},
$$

where we use \#(.) to denote the cardinality of a set. Let

$F(f, x, y, t)=\liminf _{n \rightarrow \infty} \frac{1}{n} \xi_{n}(f, x, y, t), \quad F^{*}(f, x, y, t)=\limsup _{n \rightarrow \infty} \frac{1}{n} \xi_{n}(f, x, y, t)$.

Definition 1.1. Call $x, y \in X$ a pair of points displaying strong chaos if

(1) $F(f, x, y, t)=0$ for some $t>0$,

(2) $F^{*}(f, x, y, t)=1$ for any $t>0$.

DeFinition 1.2. $f$ is said to display strong chaos if there exists an uncountable set $D \subset X$ such that any two different points in $D$ display strong chaos.

2000 Mathematics Subject Classification: 37B10, 37B40, 74H65, 34C28, 54H20.

Key words and phrases: strong chaos, topological entropy, recurrence, regular shift invariant.

Project supported by the National Science Foundation of China. 
For a continuous map $f: I \rightarrow I$, Schweizer and Smítal [8] have proved:

$\left(C_{1}\right)$ If $f$ has zero topological entropy, then no pair of points can form a strongly chaotic set.

$\left(C_{2}\right)$ If $f$ has positive entropy, then there exists an uncountable strongly chaotic set in which each member is an $\omega$-limit point of $f$.

One may pose the following questions:

$\left(Q_{1}\right)$ Is $\left(C_{1}\right)$ still true for a continuous map of any compact metric space $X$ ?

$\left(Q_{2}\right)$ Is there an uncountable strongly chaotic set in which each member is a recurrent point of $f$ on compact metric spaces?

A negative answer to $\left(Q_{1}\right)$ has been given in [6], where a minimal strongly chaotic sub-shift having zero topological entropy was constructed.

In this paper, a positive answer to $\left(Q_{2}\right)$ is given.

In fact, we will prove

Main Theorem. Let $f: X \rightarrow X$ be continuous. If $f$ has a regular shift invariant set, then it has an uncountable strongly chaotic set in which each point is recurrent, but is not almost periodic.

2. Basic definitions and preparations. Let $S=\{0,1\}, \Sigma=\{x=$ $\left.x_{1} x_{2} \ldots \mid x_{i} \in S, i=1,2, \ldots\right\}$ and define $\varrho: \Sigma \times \Sigma \rightarrow \mathbb{R}$ as follows: for any $x, y \in \Sigma$, if $x=x_{1} x_{2} \ldots$ and $y=y_{1} y_{2} \ldots$, then

$$
\varrho(x, y)= \begin{cases}0 & \text { if } x=y, \\ 1 / 2^{k} & \text { if } x \neq y \text { and } k=\min \left\{n \mid x_{n} \neq y_{n}\right\}-1 .\end{cases}
$$

It is not difficult to check that $\varrho$ is a metric on $\Sigma$. The space $(\Sigma, \varrho)$ is compact and called the one-sided symbolic space on two symbols.

Define $\sigma: \Sigma \rightarrow \Sigma$ by $\sigma\left(x_{1} x_{2} \ldots\right)=x_{2} x_{3} \ldots$ for any $x=x_{1} x_{2} \ldots \in \Sigma$. Then $\sigma$ is continuous and called the shift on $\Sigma$. Call $A$ a tuple (over $S=$ $\{0,1\})$ if it is a finite sequence of elements in $S$. If $A=a_{1} a_{2} \ldots a_{m}$ where $a_{i} \in S, 1 \leq i \leq m$, then $m$ is called the length of $A$, denoted by $|A|=m$.

For an arbitrary tuple $B=b_{1} b_{2} \ldots b_{n}$, the set $[B]=\left\{x=x_{1} x_{2} \ldots \in \Sigma\right.$, $\left.x_{i}=b_{i}, 1 \leq i \leq n\right\}$ is called the cylinder generated by $B$. For any $n \geq 1$, let

$$
\mathcal{B}_{n}=\left\{\left[b_{1} \ldots b_{n}\right] \mid b_{i}=0 \text { or } 1,1 \leq i \leq n\right\} .
$$

Then the collection $\bigcup_{n=1}^{\infty} \mathcal{B}_{n}$ is a subalgebra which generates the $\sigma$-algebra of Borel subsets of $\Sigma$. Let $h: X \rightarrow \Sigma$ be a continuous map. We use $I_{[B]}$ to denote $h^{-1}[B]$ for any $[B] \in \mathcal{B}_{n}$.

Definition 2.1. Let $f: X \rightarrow X$ be continuous. A compact set $\Lambda \subset X$ is said to be a regular shift invariant set for $f$ if: 
(1) $f(\Lambda) \subset \Lambda$,

(2) there exists a continuous surjection $h: \Lambda \rightarrow \Sigma$ satisfying

(a) $\left.h \circ f\right|_{\Lambda}=\sigma \circ h$,

(b) there exists an $M>0$ such that $\sum_{[B] \in \mathcal{B}_{n}} \operatorname{diam} I_{[B]} \leq M$ for any $n \geq 1$.

Definition 2.2. $\mathcal{B}(X)$ is the $\sigma$-algebra of Borel subsets of $X$. A probability measure $\mu$ on $(X, \mathcal{B}(X))$ is an invariant measure for $f$ if $\mu\left(f^{-1}(B)\right)$ $=\mu(B)$ for any $B \in \mathcal{B}(X)$. We denote the set of all invariant measures for $f$ by $M(X, f)$.

$\mu \in M(X, f)$ is ergodic ( $f$ can then also be regarded ergodic) if the only members $B$ of $\mathcal{B}(X)$ with $f^{-1}(B)=B$ satisfy $\mu(B)=0$ or $\mu(B)=1$.

If $\mu$ is a unique member of $M(X, f)$, it must be ergodic [9]; we then say that $f$ is uniquely ergodic.

Lemma 2.1 (see [12]). Let $f: X \rightarrow X$ and $g: Y \rightarrow Y$ be continuous, where $X$ and $Y$ are compact metric spaces. If there exists a continuous surjection $h: X \rightarrow Y$ such that $g \circ h=h \circ f$, then

(1) $h(A(f))=A(g)$,

(2) $h(R(f))=R(g)$.

Lemma 2.2 (see [10] or [11]). There exists an uncountable set $\mathcal{T}$ on the one-sided symbolic space satisfying

(1) $\mathcal{T} \subset R(\sigma)-A(\sigma)$,

(2) $\left.\sigma\right|_{\mathcal{T}}$ is strongly chaotic,

(3) $\left.\sigma\right|_{\mathcal{T}}$ is uniquely ergodic.

Lemma 2.3. Let $\sigma: \Sigma \rightarrow \Sigma$ be continuous. If $\mu$ is the only invariant probability measure for $\left.\sigma\right|_{R(\sigma)-A(\sigma)}$, then $\mu(\{x\})=0$ for any $x \in R(\sigma)-A(\sigma)$.

Proof. Let $x \in R(\sigma)-A(\sigma)$. We first claim that $\{x\}, \sigma^{-1}(x), \sigma^{-2}(x), \ldots$ are pairwise disjoint. Assume the claim to be false; then $\sigma^{-m}(x) \cap \sigma^{-n}(x)$ $\neq \emptyset$ for some $m$ and $n$ with $m>n \geq 0$. Take $y \in \sigma^{-m}(x) \cap \sigma^{-n}(x)$, so $\sigma^{m}(y)=\sigma^{n}(y)=x$. Furthermore,

$$
\sigma^{m-n}(x)=\sigma^{m-n}\left(\sigma^{n}(y)\right)=\sigma^{m}(y)=x,
$$

i.e. $x$ is a periodic point, which contradicts $x \in R(\sigma)-A(\sigma)$. Since $\mu$ is an invariant probability measure for $\left.\sigma\right|_{R(\sigma)-A(\sigma)}$ and the set of simple points on $(\Sigma, \varrho)$ is closed, we have $\{x\} \in \mathcal{B}(\Sigma)$ and

$$
\mu(\{x\})=\mu\left(\sigma^{-1}(x)\right)=\mu\left(\sigma^{-2}(x)\right)=\ldots=\mu\left(\sigma^{-n}(x)\right) .
$$

By the countable additivity of $\mu$, we get $\mu(\{x\})=0$. 
Lemma 2.4. Suppose $\mathcal{T}=R(\sigma)-A(\sigma)$. If $\mu$ is the only invariant probability measure for $\left.\sigma\right|_{\mathcal{T}}$, then the sequence $\left\{\mu\left(\left[b_{1} \ldots b_{n}\right]\right)\right\}$ of real numbers converges to zero uniformly in $b_{i} \in\{0,1\}, 1 \leq i \leq n$, as $n \rightarrow \infty$.

Proof. For any $\varepsilon>0$ and any $x \in \mathcal{T}$, by Lemma 2.3, there is an open neighborhood $V_{x}$ of $x$ such that $\mu\left(V_{x}\right)<\varepsilon$. Moreover, by the definition of $\left[b_{1} \ldots b_{n}\right]$, there exists $N>0$ such that $\operatorname{diam}\left[b_{1} \ldots b_{n}\right]<\varepsilon$ uniformly in $b_{i} \in\{0,1\}, 1 \leq i \leq n$, as $n \rightarrow \infty$. Thus for any $x \in\left[b_{1} \ldots b_{n}\right] \cap \mathcal{T}$, there exists $N>0$ such that $x$ must be contained in some $V_{x}$ when $n \geq N$. So

$$
\mu\left(\left[b_{1} \ldots b_{n}\right]\right)=\mu\left(\left[b_{1} \ldots b_{n}\right] \cap \mathcal{T}\right)<\varepsilon .
$$

Lemma 2.5 (see [7]). Let $f: X \rightarrow X$ be continuous, $x, y \in X, N>0$.

(1) If $F\left(f^{N}, x, y, s\right)=0$ for any $s>0$, then there exists a $t>0$ such that $F(f, x, y, t)=0$.

(2) If $F^{*}\left(f^{N}, x, y, s\right)=1$ for any $s>0$, then $F^{*}(f, x, y, t)=1$ for any $t>0$.

3. Proof of the main theorem. By the hypothesis, $f$ has a regular shift invariant set, denoted by $\Lambda$. Thus there is a continuous surjection $h$ : $\Lambda \rightarrow \Sigma$ such that for any $x \in \Lambda$,

$$
h \circ f(x)=\sigma \circ h(x) .
$$

According to Lemma 2.2, there is an uncountable set $\mathcal{T} \subset R(\sigma)-A(\sigma)$ which is strongly chaotic and $\left.\sigma\right|_{\mathcal{T}}$ has the only ergodic measure $\mu$. Set, for simplicity, $g=\left.f\right|_{\Lambda}$. For any $y \in \mathcal{T}$, by Lemma 2.1 there exists $x \in$ $R(g)-A(g)$ such that $h(x)=y$. Let

$$
D=\{x \mid x \in R(g)-A(g), h(x)=y \text { and } y \in \mathcal{T}\} .
$$

Then $D \subset \Lambda$ and $D$ is an uncountable set. To complete the proof, it suffices to show that $D$ is a strongly chaotic set for $f$.

For any distinct $x_{1}, x_{2} \in D$, there exist $y_{1}, y_{2} \in \mathcal{T}$ such that $h\left(x_{i}\right)=y_{i}$ for $i=1,2$. Since $y_{1}$ and $y_{2}$ are in a strongly chaotic set for $\sigma$, there exists $s>0$ and a sequence $n_{k} \rightarrow \infty$ such that

$$
\frac{1}{n_{k}} \xi_{n_{k}}\left(\sigma, y_{1}, y_{2}, s\right) \rightarrow 0 \quad(k \rightarrow \infty) .
$$

Choose an $N>0$ such that $\operatorname{diam}[B]<s$ for any $[B] \in \mathcal{B}_{N}$. Let

$$
t=\min \left\{d\left(I_{[B]}, I_{[C]}\right) \mid[B],[C] \in \mathcal{B}_{N} \text { and }[B] \neq[C]\right\},
$$

where $d\left(I_{[B]}, I_{[C]}\right)=\inf \left\{d(p, q) \mid p \in I_{[B]}, q \in I_{[C]}\right\}$. By the properties of $g$, $d\left(I_{[B]}, I_{[C]}\right)>0$ for any distinct $[B],[C] \in \mathcal{B}_{N}$ and so $t>0$. It is easily seen that for any $i \geq 0$, 


$$
\begin{aligned}
\varrho\left(\sigma^{i}\left(y_{1}\right), \sigma^{i}\left(y_{2}\right)\right) & \geq s \\
\Rightarrow \sigma^{i}\left(y_{1}\right) & \in[B], \sigma^{i}\left(y_{2}\right) \in[C]
\end{aligned}
$$

for some distinct $[B],[C] \in \mathcal{B}_{N}$ (since $\operatorname{diam}[B]<s$ )

$$
\begin{aligned}
& \Rightarrow g^{i}\left(x_{1}\right) \in I_{[B]}, g^{i}\left(x_{2}\right) \in I_{[C]} \text { and } d\left(I_{[B]}, I_{[C]}\right) \geq t \\
& \Rightarrow d\left(g^{i}\left(x_{1}\right), g^{i}\left(x_{2}\right)\right) \geq t
\end{aligned}
$$

and therefore, for each $k$ we have

$$
\xi_{n_{k}}\left(g, x_{1}, x_{2}, t\right) \leq \xi_{n_{k}}\left(\sigma, y_{1}, y_{2}, s\right) .
$$

By (3.1), we get

and hence

$$
\frac{1}{n_{k}} \xi_{n_{k}}\left(g, x_{1}, x_{2}, t\right) \rightarrow 0 \quad(k \rightarrow \infty),
$$

$$
F\left(g, x_{1}, x_{2}, t\right)=0 .
$$

We now prove $F^{*}\left(g, x_{1}, x_{2}, t\right)=1$ for any $t>0$. By the hypothesis, we can choose $M>0$ such that $\sum_{[B] \in \mathcal{B}_{n}} \operatorname{diam} I_{[B]} \leq M$ for any fixed $n>0$. For any given $t>0$ and $\varepsilon>0$, choose an integer $k>0$ such that $t k>M$. By Lemma 2.4, we may also choose an $N_{1}$ large enough such that $\mu([B])<$ $\varepsilon /(2 k)$ for any $[B] \in \mathcal{B}_{N_{1}} \cap \mathcal{T}$, i.e. for any $y \in \mathcal{T}$,

$$
\lim _{n \rightarrow \infty} \frac{1}{n} \#\left\{i \mid \sigma^{i}(y) \in[B], 0 \leq i \leq n\right\}<\frac{\varepsilon}{2 k} .
$$

Put $s=1 / 2^{N_{1}}$. Since $F^{*}\left(\sigma, y_{1}, y_{2}, s\right)=1$, there exists a sequence $n_{j} \rightarrow \infty$ such that

$$
\frac{1}{n_{j}} \xi_{n_{j}}\left(\sigma, y_{1}, y_{2}, s\right) \rightarrow 1 \quad\left(n_{j} \rightarrow \infty\right) .
$$

Set, for simplicity,

$$
\theta_{n_{j}}=\sum_{[B] \in \mathcal{B}_{N_{1}} \cap \mathcal{T}} \frac{1}{n_{j}} \#\left\{i \mid g^{i}\left(x_{1}\right), g^{i}\left(x_{2}\right) \in I_{[B]}, 0 \leq i \leq n_{j}\right\} .
$$

Noting that

$$
\begin{aligned}
\varrho\left(\sigma^{i}\left(y_{1}\right), \sigma^{i}\left(y_{2}\right)\right) & <s \\
& \Leftrightarrow \sigma^{i}\left(y_{1}\right), \sigma^{i}\left(y_{2}\right) \in[B] \text { for some }[B] \in \mathcal{B}_{N_{1}} \cap \mathcal{T} \\
& \Leftrightarrow g^{i}\left(x_{1}\right), g^{i}\left(x_{2}\right) \in I_{[B]} \text { for some }[B] \in \mathcal{B}_{N_{1}} \cap \mathcal{T},
\end{aligned}
$$

according to (3.4), we have

$$
\theta_{n_{j}} \rightarrow 1 \quad(j \rightarrow \infty) .
$$

Thus from (3.3), (3.5), and (3.6) we can choose $N$ large enough such that for $n_{j}>N$, 
$\frac{1}{n_{j}} \#\left\{i \mid g^{i}\left(x_{1}\right), g^{i}\left(x_{2}\right) \in I_{[B]}, 0 \leq i<n_{j}\right\}<\frac{\varepsilon}{2 k} \quad$ for any $[B] \in \mathcal{B}_{N_{1}} \cap \mathcal{T}$ and

$$
1-\theta_{n_{j}}<\varepsilon / 2
$$

On the one hand, by the definition of $\theta_{n_{j}}$,

$$
\begin{aligned}
\theta_{n_{j}}- & \sum_{\substack{[B] \in \mathcal{B}_{N_{1}} \cap \mathcal{T} \\
\operatorname{diam} I_{[B]} \geq t}} \frac{\varepsilon}{2 k} \\
\leq & \theta_{n_{j}}-\sum_{\substack{[B] \in \mathcal{B}_{N_{1}} \cap \mathcal{T} \\
\operatorname{diam} I_{[B]} \geq t}} \frac{1}{n_{j}} \#\left\{i \mid g^{i}\left(x_{1}\right), g^{i}\left(x_{2}\right) \in I_{[B]}, 0 \leq i<n_{j}\right\} \\
= & \sum_{\substack{[B] \in \mathcal{B}_{N_{1}} \cap \mathcal{T} \\
\operatorname{diam} I_{[B]}<t}} \frac{1}{n_{j}} \#\left\{i \mid g^{i}\left(x_{1}\right), g^{i}\left(x_{2}\right) \in I_{[B]}, 0 \leq i<n_{j}\right\} \\
\leq & \frac{1}{n_{j}} \xi_{n_{j}}\left(g, x_{1}, x_{2}, t\right) .
\end{aligned}
$$

On the other hand, because of the choice of $k$, there exist at most $k$ different $[B]$ 's with $\operatorname{diam} I_{[B]} \geq t$ in $\mathcal{B}_{N_{1}} \cap \mathcal{T}$. In fact, since $t k>M$, if there exists $k_{1}>k$ such that $k_{1}$ different $[B]$ 's satisfy diam $I_{[B]} \geq t$, then $k_{1} \operatorname{diam} I_{[B]} \geq$ $t k_{1}>M$. However, by the choice of $M$, we know that

$$
M \geq \sum_{[B] \in \mathcal{B}_{N_{1}} \cap \mathcal{T}} \operatorname{diam} I_{[B]} \geq k_{1} \operatorname{diam} I_{[B]},
$$

which is contradictory. By (3.8), we have

$$
\theta_{n_{j}}-\frac{\varepsilon}{2}=\theta_{n_{j}}-k \cdot \frac{\varepsilon}{2 k} \leq \frac{1}{n_{j}} \xi_{n_{j}}\left(g, x_{1}, x_{2}, t\right) .
$$

Combining this with (3.7), we see that for $n_{j}>N$,

$$
0 \leq 1-\frac{1}{n_{j}} \xi_{n_{j}}\left(g, x_{1}, x_{2}, t\right) \leq 1-\theta_{n_{j}}+\frac{\varepsilon}{2}<\frac{\varepsilon}{2}+\frac{\varepsilon}{2}=\varepsilon,
$$

which gives

$$
F^{*}\left(g, x_{1}, x_{2}, t\right)=1
$$

By (3.2), (3.9) and the arbitrariness of $x_{1}$ and $x_{2}$, we conclude that $D$ is an uncountable strongly chaotic set of $g$.

Thus, we have proved that $f$ has an uncountable strongly chaotic set $D$ in $R(f)-A(f)$. 


\section{Examples}

EXAMPLE 4.1. Let $f \in C^{0}(I)$. If $f$ has a positive topological entropy, then there exists an $N>0$ such that $f^{N}$ has a regular shift invariant set ([1]). From the theorem, we deduce that $f^{N}$ has an uncountable strongly chaotic set in which each point is recurrent, but is not almost periodic. The same holds for $f$, since for any positive integer $n, f$ displays strong chaos if and only if $f^{n}$ does (Lemma 2.5).

EXAMPLE 4.2. Let $r_{0}, r_{1}: S^{1} \rightarrow S^{1}$ be irrational rotations with $r_{0} \neq \pm r_{1}$. Define $f: \Sigma \times S^{1} \rightarrow \Sigma$ by

$$
f(x, t)=\left(\sigma(x), r_{x_{1}}(t)\right)
$$

for $x=x_{1} x_{2} \ldots \in \Sigma, t \in S^{1}$. Note that the $n$th iteration of $f$ at the point $(x, t) \in \Sigma \times S^{1}$ is given by

$$
f^{n}(x, t)=\left(\sigma^{n}(x), r_{x_{n}} \circ \ldots \circ r_{x_{2}} \circ r_{x_{1}}(t)\right) .
$$

It is easy to see that $f$ is continuous.

Let $h: \Sigma \times S^{1} \rightarrow \Sigma$ be defined by $h(x, t)=x$. We see that $h$ satisfies (2)(a) of Definition 2.1, but not (2)(b). Indeed, we do not know if an $h$ satisfying both (2)(a) and (2)(b) exists or not. Also we do not know if $f$ displays strong chaos or not, since our theorem cannot be used.

\section{References}

[1] L. S. Block and W. A. Coppel, Dynamics in One Dimension, Springer, New York, 1992, 35-36.

[2] B. S. Du, Every chaotic interval map has a scrambled set in the recurrent set, Bull. Austral. Math. Soc. 39 (1989), 259-264.

[3] T. Y. Li, M. Misiurewicz, G. Pianigiani and J. Yorke, Odd chaos, Phys. Lett. A 87 (1982), 271-273.

[4] - - - - - , No division implies chaos, Trans. Amer. Math. Soc. 273 (1982), 191-199.

[5] G. F. Liao, Chain recurrent orbits of mapping of the interval, Northeast. Math. J. 2 (1986), 240-244.

[6] G. F. Liao and Q. J. Fan, Minimal subshifts which display Schweizer-Smital chaos and have zero topological entropy, Sci. China Ser. A 41 (1998), 33-38.

[7] G. F. Liao and L. D. Wang, Almost periodicity and SS scrambled sets, Chinese Ann. Math. Ser. A 23 (2002), 685-692 (in Chinese).

[8] B. Schweizer and J. Smítal, Measures of chaos and a spectral decomposition of dynamical systems on the interval, Trans. Amer. Math. Soc. 344 (1994), 737-754.

[9] P. Walters, An Introduction to Ergodic Theory, Springer, New York, 1982.

[10] L. D. Wang, Z. Y. Chu and G. F. Liao, Recurrence, strong chaos and unique ergodicity, Jilin Shifan Daxue Xuebao 82 (2003), 11-15 (in Chinese).

[11] L. D. Wang, G. F. Liao and X. Yang, Recurrent point set of the shift on $\Sigma$ and strong chaos, Ann. Polon. Math. 78 (2002), 123-130. 
[12] J. C. Xiong, Set of almost periodic points of a continuous self-map of an interval, Acta Math. Sinica 2 (1986), 73-77.

[13] R. S. Yang, Pseudo-shift-invariant sets and chaos, Chinese Ann. Math. Ser. A 13 (1992), 22-25 (in Chinese).

[14] Z. L. Zhou and W. H. He, Level of the orbit's structure and topological semiconjugacy, Sci. China Ser. A 38 (1995), 897-907.

Lidong Wang and Xiaodong Duan

Research Institute of Nonlinear Information Technology

Dalian Nationalities University

Dalian 116600, P.R. China

E-mail: wldwyylfx@hotmail.com

Zhizhi Chen

Graduate Department of Jilin Normal University

Siping 136000, P.R. China

E-mail: dingding_chen@263.sina.com
Gongfu Liao

Institute of Mathematics

Jilin University

Changchun 130023, P.R. China

E-mail: liaogf@public.cc.jl.cn

Reçu par la Rédaction le 13.3.2003

Révisé le 20.6.2003 